\title{
A monocarboxylate transporter required for hepatocyte secretion of ketone bodies during fasting
}

\author{
Sarah E. Hugo, ${ }^{1,2}$ Lourdes Cruz-Garcia,, ${ }^{1,2}$ Santhosh Karanth, ${ }^{1,2}$ Ryan M. Anderson,, \\ Didier Y.R. Stainier, ${ }^{3}$ and Amnon Schlegel ${ }^{1,2,4,6}$ \\ ${ }^{1}$ University of Utah Molecular Medicine (U2M2) Program, ${ }^{2}$ Department of Internal Medicine, Division of Endocrinology, \\ Metabolism, and Diabetes, University of Utah, Salt Lake City, Utah 84112, USA; ${ }^{3}$ Department of Biochemistry and Biophysics, \\ Liver Center, Diabetes Center, Cardiovascular Research Institute, University of California at San Francisco, San Francisco, \\ California 94158, USA; ${ }^{4}$ Department of Biochemistry, School of Medicine, University of Utah, Salt Lake City, Utah 84112, USA
}

To find new genes that influence liver lipid mass, we performed a genetic screen for zebrafish mutants with hepatic steatosis, a pathological accumulation of fat. The red moon (rmn) mutant develops hepatic steatosis as maternally deposited yolk is depleted. Conversely, hepatic steatosis is suppressed in rmn mutants by adequate nutrition. Adult rmn mutants show increased liver neutral lipids and induction of hepatic lipid biosynthetic genes when fasted. Positional cloning of the rmn locus reveals a loss-of-function mutation in slc16aba (solute carrier family 16a, member 6a), a gene that we show encodes a transporter of the major ketone body $\beta$-hydroxybutyrate. Restoring wild-type zebrafish slc16aba expression or introducing human SLC16A6 in rmn mutant livers rescues the mutant phenotype. Radiotracer analysis confirms that loss of Slc16a6a function causes diversion of livertrapped ketogenic precursors into triacylglycerol. Underscoring the importance of Slc16a6a to normal fasting physiology, previously fed rmn mutants are more sensitive to death by starvation than are wild-type larvae. Our unbiased, forward genetic approach has found a heretofore unrecognized critical step in fasting energy metabolism: hepatic ketone body transport. Since $\beta$-hydroxybutyrate is both a major fuel and a signaling molecule in fasting, the discovery of this transporter provides a new direction for modulating circulating levels of ketone bodies in metabolic diseases.

[Keywords: hepatic steatosis; ketone body; lipid metabolism; starvation; zebrafish]

Supplemental material is available for this article.

Received October 13, 2011; revised version accepted December 27, 2011.

Hepatic steatosis is present in a large fraction of obese people (Clark 2006). Nonalcoholic fatty liver disease (NAFLD) encompasses hepatic steatosis and several pathological states that follow it: inflammation (steatohepatitis), fibrosis (cirrhosis), and cancer (hepatocellular carcinoma) (Liou and Kowdley 2006). There are limited therapeutic options for permanently ameliorating hepatic steatosis (Sanyal et al. 2010), and there are no methods for reversing hepatic fibrosis or preventing hepatocellular carcinoma due to NAFLD (Nugent and Younossi 2007). The first step of NAFLD is the inappropriate accumulation of triacylglycerol in hepatocytes (Browning and Horton 2004). This accumulation may be due to excessive de novo hepatic lipid production; decreased hepatic secretion of very low-

\footnotetext{
${ }^{5}$ Present address: Department of Pediatrics, Indiana University School of Medicine, 635 Barnhill Drive, MS2053, Indianapolis, IN 46202, USA. ${ }^{6}$ Corresponding author.

E-mail amnons@u2m2.utah.edu.

Article is online at http://www.genesdev.org/cgi/doi/10.1101/gad.180968.111.
}

density lipoprotein (VLDL) particles; diminished $\beta$-oxidation of fatty acids in the liver; exaggerated uptake of fatty acids from the circulation; more subtle defects in regulating energy homeostasis, including insulin resistance or CNS nutrient sensing; or a combination of these factors (Hooper et al. 2011). Since each of these processes could be exploited therapeutically, understanding their regulation is paramount. Identifying and characterizing novel genes that modulate hepatic lipid mass are priorities /Cohen et al. 2011).

Master transcription factors, metabolic regulators, and multiple enzymes that control lipid homeostasis are present in zebrafish (Babin and Vernier 1989; Ibabe et al. 2002; Schlombs et al. 2003). This conservation of function and the absence of fasting steatosis in larvae that have never been fed (Schlegel and Stainier 2006) allowed us to screen for mutations causing increased hepatic lipid mass. Performing the screen in animals carrying three transgenes that encode fluorescent protein labels of the Islet of Langerhans $\beta$ cells, the liver hepatocytes, and the pancreatic 
acinar cells (Anderson et al. 2009) helped us to avoid mutants that displayed changes in liver or pancreas morphology (Sadler et al. 2005; Akimitsu et al. 2008; Matthews et al. 2009; Thakur et al. 2011). Defects in yolk lipid transport were avoided by restricting our analysis to only larvae that consumed all their yolk by $6 \mathrm{~d}$ post-fertilization (dpf) (Schlegel and Stainier 2006; Raldúa et al. 2008; Walters et al. 2009). Similarly, animals that showed craniofacial malformations (Schlombs et al. 2003; Sadler et al. 2005) and overt neuronal defects were excluded because we felt they would not be amenable to characterization beyond the early larval period and because they often show residual yolk lipids at $6 \mathrm{dpf}$.

Here we report the identification, positional cloning, and functional characterization of one mutant that reveals a heretofore unappreciated bottleneck of hepatic lipid metabolism: ketone body transport.

\section{Results}

Isolation and initial characterization of red moon (rmn), a zebrafish mutant with hepatic steatosis

The rmn mutant showed hepatic steatosis just as maternally supplied yolk lipids were nearing exhaustion on $5 \mathrm{dpf}$ (Fig. 1A). Aside from the surfactant-lined swim bladder, only the rmn mutant liver showed strong Oil Red O (ORO) staining on 6 and $7 \mathrm{dpf}$. The accumulation of neutral lipids in rmn mutant livers was confirmed with both wholemount and confocal microscopy of animals stained with the fluorescent neutral lipid dye 4,4-difluoro-1,3,5,7,8pentamethyl-4-bora-3a,4a-diaza-s-indacene (BODIPY 493/ 503) (Fig. 1B,C). Numerous cytoplasmic lipid droplets in otherwise normal hepatocytes were observed in rmn mutants examined with transmission electron microscopy (Fig. 1D). Normal macronutrient recycling was appreciated in rmn livers viewed at higher magnification (Fig. 1D). Similarly, histological analysis demonstrated no evidence of inflammatory cell infiltration or deposition of excessive extracellular matrix proteins (Supplemental Fig. S1A,B). Taken together, the histological and ultrastructural characterization indicated that our stringent screening criteria were met: The fulminant hepatic degeneration seen in other zebrafish mutants with hepatic steatosis did not occur in rmn mutants.

The lack of secondary phenotypes in rmn mutants enabled us to assess whether the steatosis phenotype would be modulated by prolonged fasting. Hepatic steatosis in rmn mutants persisted in the never-fed state to 12 dpf in the absence of other, overt morphological defects (Supplemental Fig. S1C). This persistence of hepatic steatosis prompted us to quantify the major neutral lipid species in whole-larval extracts over the course of a prolonged fast. There were increased levels of triacylglycerol, $\beta$-hydroxybutyrate, and free fatty acids in whole-larval extracts $5 \mathrm{dpf}$ (Fig. 2A). The lipid compositions of wild-type and rmn mutant larvae changed over the course of the fast. Cholesterol was the most abundant neutral lipid in wholelarval extracts and was more abundant in rmn mutant extracts on 7 and $8 \mathrm{dpf}$. Similar to $\beta$-hydroxybutyrate and
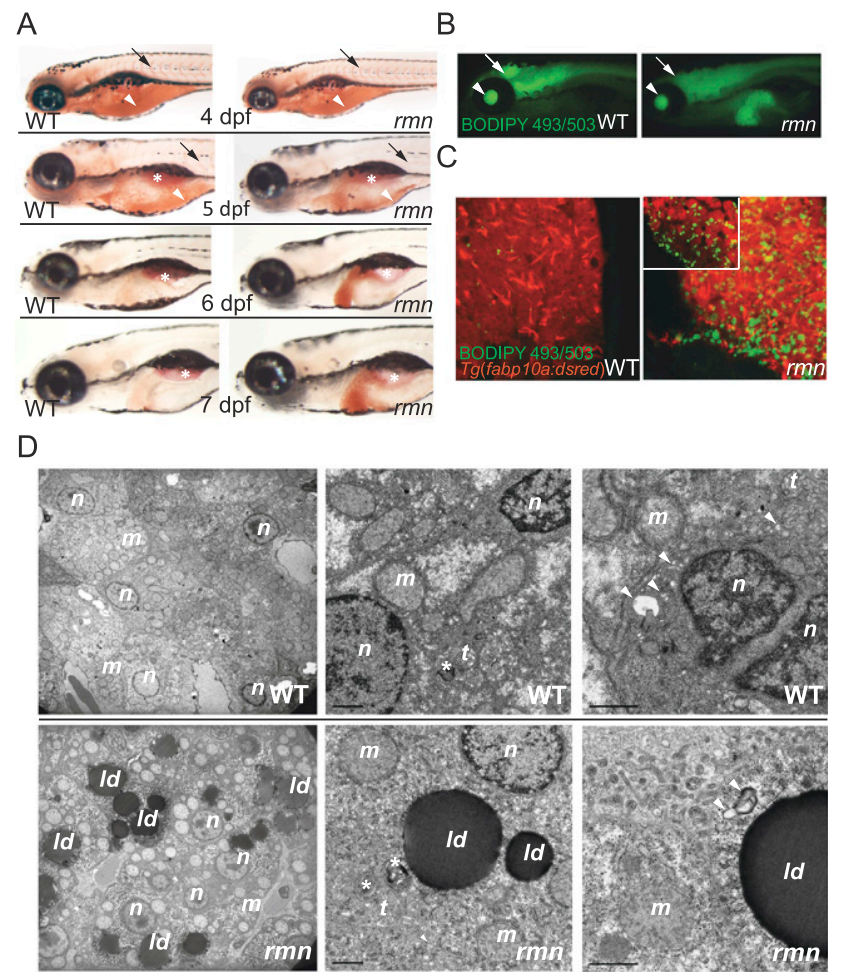

Figure 1. Identification of the fasting hepatic steatosis mutant rmn. (A) Whole-mount ORO staining of larvae. The yolk lipid (arrowhead) is exhausted 5 dpf. Vascular lipid staining (arrows) ceases by the end of $5 \mathrm{dpf}$. The surfactant-lined swim bladder stained with ORO (asterisk). (B) Whole-mount BODIPY 493/503 staining of 6-dpf larvae. This dye also stained the vitreous humor of the eyes (arrowhead) and the ventricles of the CNS (arrow). (C) Confocal stacks of livers fixed and stained as in $B$. A single slice of a rmn mutant liver is shown in the inset. $(D)$ Transmission electron microscopy of liver sections showing that the rmn mutant hepatocytes have cytoplasmic lipid droplets (ld). The nuclear (n) and mitochondrial $(\mathrm{m})$ morphology appears normal in rmn mutants. Higher-magnification micrographs also show multilamellar structures (asterisks) suggestive of multivesicular bodies and elongated tubular $(\mathrm{t})$ precursors of these structures in both wild-type (WT) and the rmn mutant livers. Similarly, autophagosomal structures (arrowheads) were observed in both wild-type and $r m n$ mutant livers. Bar, $1 \mu \mathrm{m}$.

free fatty acids, the triacylglycerol was also higher in 5- and 6-dpf rmn mutant extracts. Cholesteryl esters were more abundant in rmn mutants between 7 and $10 \mathrm{dpf}$. Thereafter, the whole-larval levels of all three neutral lipids were not different. In summary, never-fed rmn mutants showed persisting hepatic steatosis marked by accumulation of different neutral lipid species until death by $13 \mathrm{dpf}$.

Since rmn mutant larvae showed no obvious morphological phenotypes, it was possible that the mutation was not lethal. We raised an in-cross of heterozygous carriers under normal feeding conditions and observed that the rmn mutant was viable /recovered in the anticipated Mendelian ratio of a fully penetrant and fully expressive recessive mutation) and fertile. Thus, we were able to assess the changes in hepatic neutral lipid composition by subjecting wild-type and rmn mutant adult animals to a 
Hugo et al.
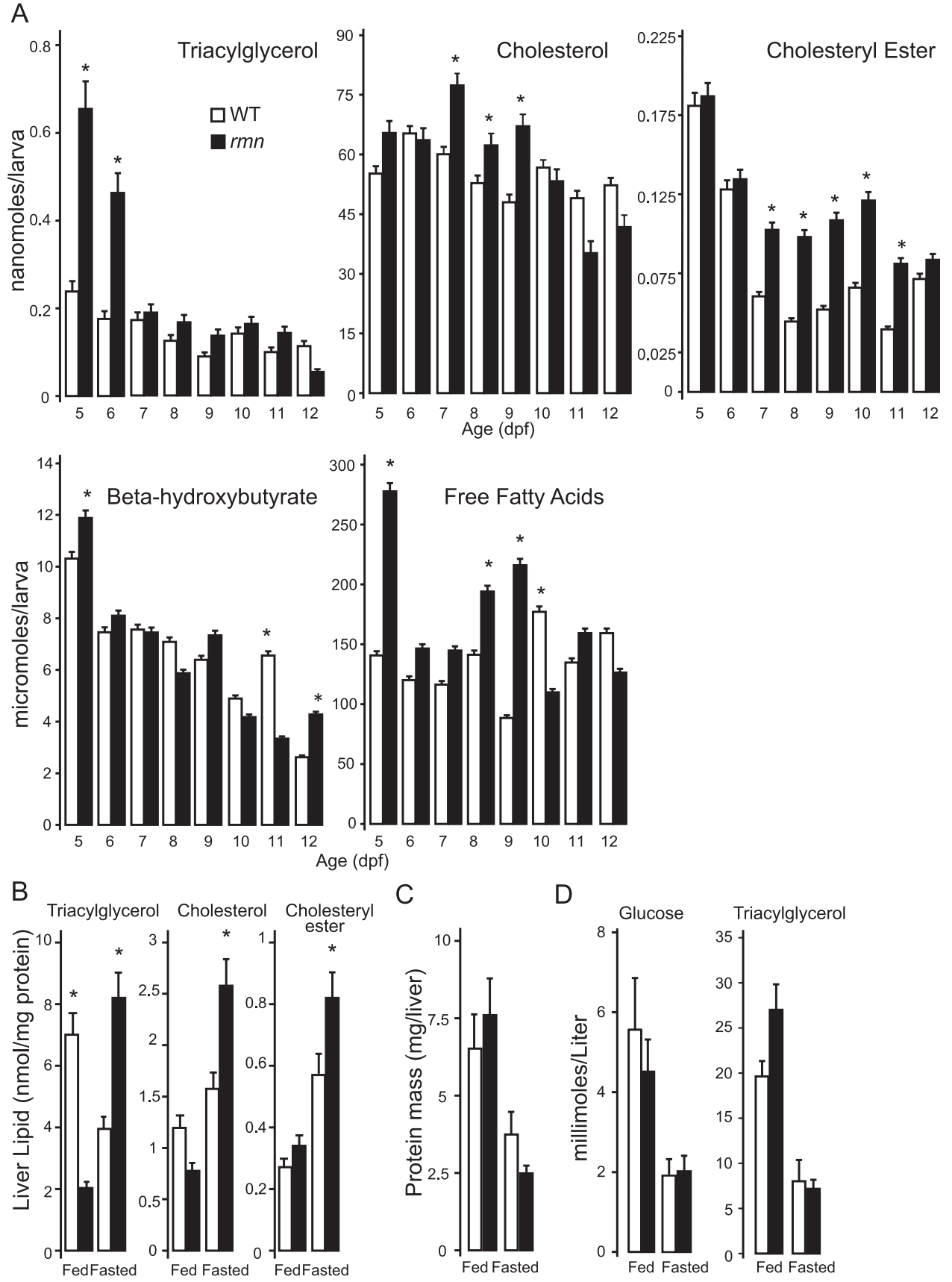

Figure 2. Increased neutral lipids in rmn mutants. (A) $\beta$-Hydroxybutyrate $(n=10$ larvae for each genotype), cholesterol $(n=15)$, cholesteryl esters $(n=50)$, free fatty acids $(n=40)$, and triacylglycerol $(n=50)$ measured in whole-body extracts of larvae. $\left(^{\star}\right) P<0.01$ for the age-matched comparator. $(B)$ Neutral lipid levels in fed and fasted adult livers. $\left({ }^{\star}\right) P<0.01$ for the nutritional status comparator $(n=4)$. $(C)$ Adult liver protein mass in fed and fasted animals $(n=4)$. $(D)$ Blood glucose and triacylglycerol from fed and fasted animals. $\left(^{\star}\right) P<0.01$ for the age-matched comparator $(n=4)$. In all panels, wild-type is shown in open bars, and rmn mutant is solid bars.

2-wk fast and analyzing their dissected livers. Recapitulating the larval phenotype, fasted rmn mutants showed increased levels of triacylglycerol, cholesterol, and cholesteryl esters (Fig. 2B). Importantly, this experimental approach led to equal protein mass loss in fasted wild-type and rmn mutant livers (Fig. 2C). Validating our experimental design, we observed that wild-type and $\mathrm{rmn}$ mutant adults were hypoglycemic and had lower blood triacylglycerol concentrations when fasted (Fig. 3D). The adult rmn livers showed modest microvesicular steatosis without evidence of inflammation (Supplemental Fig. S2). Intrahepatic $\beta$-hydroxybutyrate was slightly lower in fasting rmn livers (Supplemental Fig. S3A). The serum $\beta$-hydroxybutyrate did not rise during the fasting period, even in wild-type animals (Supplemental Fig. S3B). 
A

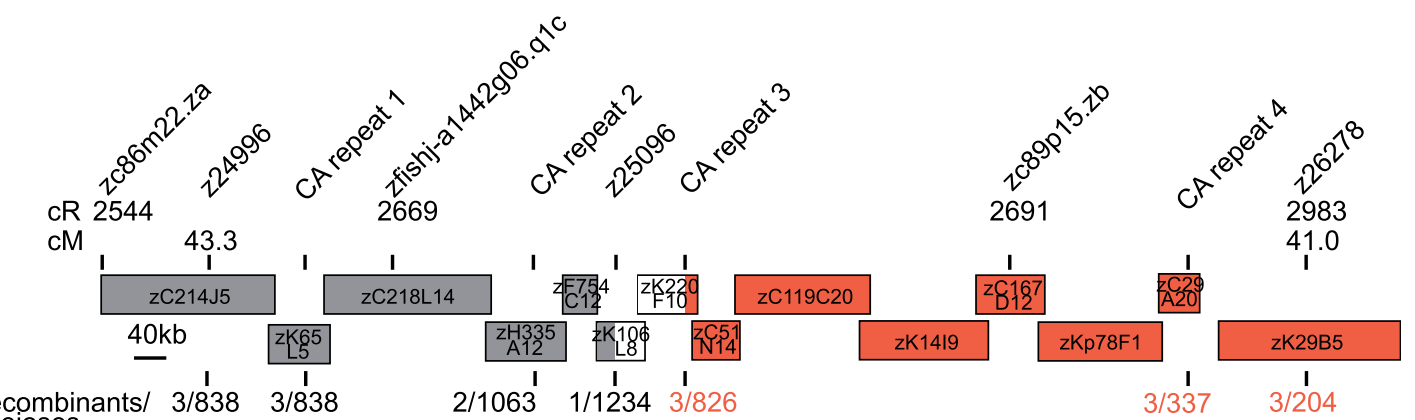
meioses

2/1063 $1 / 1234 \quad 3 / 826$

B recombinants/
meioses

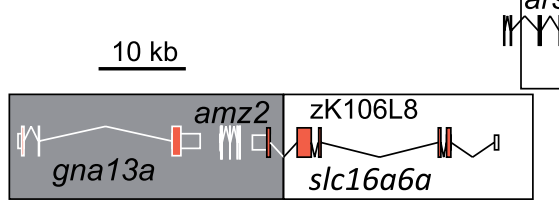

C

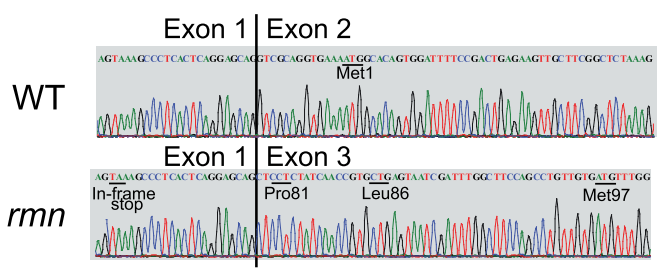

D

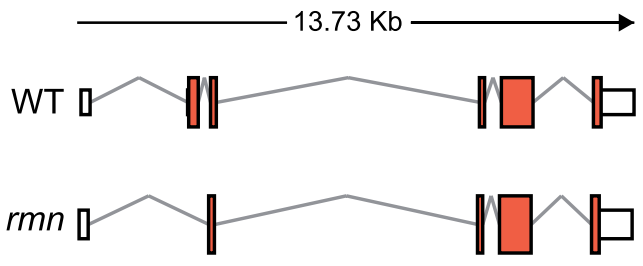

E

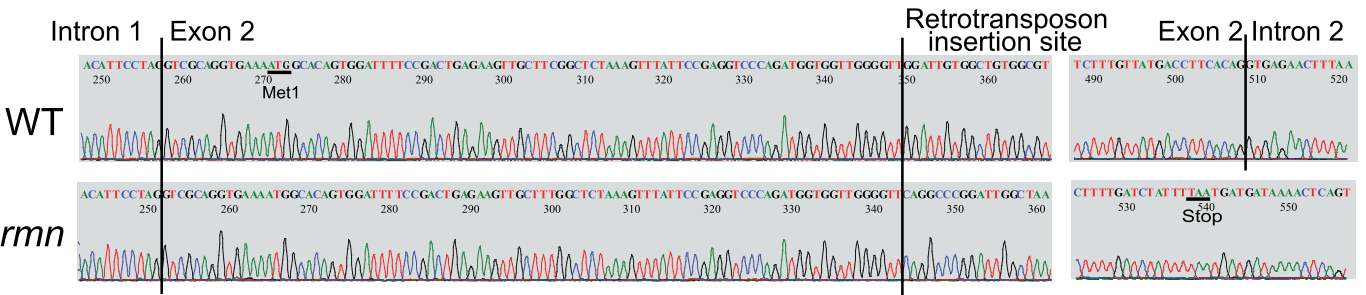

F

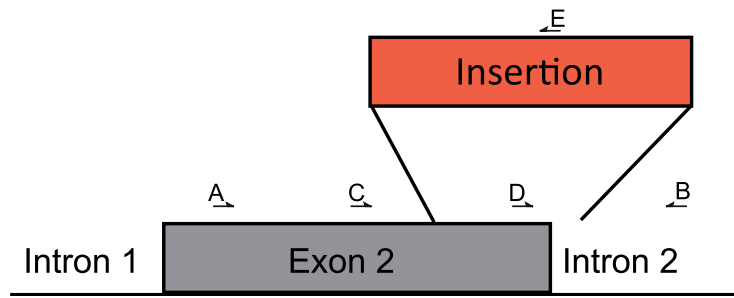

G

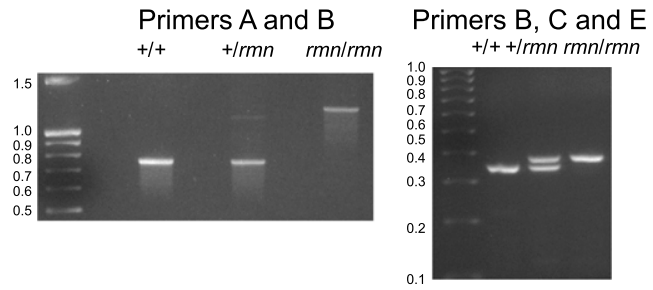

Figure 3. Positional cloning of the rmn locus. (A) The rmn locus was narrowed to a 100 -kb critical interval on chromosome 12 . Genetic distance of markers is reported on the radiation hybrid map in centiRays (cR) and on the MGH meiotic map in centiMorgans (cM) above the individual bacterial artificial chromosome and fosmid clones used to span this region of the genome. The recombination events (reported as recombinants/meioses) at the labeled polymorphic markers (poly-CA or "CA repeat") are shown below individual clones. Clone acronym definitions and the names of the clones between zK29B5 and zC29A20 are in the Supplemental Material. $(B)$ Fine mapping narrowed the critical interval to the space between two CA repeats within introns of slc16a6a and calcoco2 on the clone zK106L8 (white region flanked by gray and red portions of the chromosome). Coding exons are filled red boxes. The 5' and 3' untranslated regions (UTRs) are unfilled boxes. (C) Sanger traces of slc16a6a cDNAs prepared with primers in the $5^{\prime}$ and $3^{\prime}$ UTRs indicating that exon 2 is skipped in rmn mutants. The position of leucine codon 86 and methionine codon 97 are shown. (D) Cartoons of slc16a6a cDNAs prepared as in $C$. (E) Sanger traces of genomic DNA spanning exon 2 of slc16a6a reveal retrotransposon sequences in rmn mutants. An in-frame stop codon is shown. $(F)$ Cartoon of the insertion mutation destroying exon 2 of slc16a6a. $(G$, left $)$ The sequenced products shown in $E$ differ in size: Amplification of exon 2 and flanking intronic sequences of slc16a6a from homozygous wild-type animals $(+/+)$, heterozygous $r m n$ carriers $(+/$ rmn $)$, and homozygous $r m n$ mutant animals $(\mathrm{rmn} / \mathrm{rmn})$ reveals a 450-bp increase in the rmn allele product. (Left panel) Heterozygous carriers proved difficult to score using this primer pair because the mutant allele amplified less robustly in the presence of the wild-type allele (primers A and B). (Right panel) Thus, we resorted to a three-primer strategy to amplify either the sequences deleted by the insertion or a small portion of the insertion. The latter approach led to confident scoring of heterozygous carriers. The molecular mass standards are shown in kilobase pairs. 
The rmn mutation disrupts an orphan monocarboxylate transporter (MCT) gene

Positional cloning methods were used to isolate the gene disrupted by the rmn mutation (Fig. 3A,B). Of the genes within the critical interval on chromosome 12, slc16a6a (solute carrier family $16 a$, member $6 a$ ) was a strong candidate because it encodes an orphan MCT that may be required in the transport of nutrients as animals transition from fed to fasted states (Price et al. 1998). We cloned and sequenced mature slc16a6a cDNAs from adult liver and observed aberrant mRNA maturation with skipping of the initiator ATG codon-containing exon 2 in rmn mutants (Fig. 3C,D). In rmn mutants, the 3' 169 base pairs (bp) of exon 2 and the first $67 \mathrm{bp}$ of intron 2 in the slc16a6a gene were replaced by a 404-bp sequence that is most similar to the DrNgaro4 retrotransposon (Fig. 3E-G; Supplemental Fig. S4; Goodwin and Poulter 2004). Thus, the rmn mutation was not induced by $N$-ethyl- $N$-nitrosourea. Rather, it was carried in some of the mutagenized animals we used for the screen (Anderson et al. 2009). The rmn mutation was found in other strains (Supplemental Material), but not in the one we used in our previous studies (Schlegel and Stainier 2006).

In contrast to the nearly ubiquitous expression of Slc16a1 (MCT1) and Slc16a7 (MCT2), the expression of Slc16a6 is limited to liver, pancreas, skin, vas deferens, and testis of adult rats (Bonen et al. 2006). The expression pattern of Slc16a6 during development is not known. The zebrafish slc16a6a transcript was not maternally deposited, but was detected throughout the embryonic and early larval period (Fig. 4A). Whole-mount in situ hybridization revealed expression of slc16a6a in the head and pronephros early in development and increased expression in the liver, proximal intestine, and swim bladder by $5 \mathrm{dpf}$ (Fig. 4B).

Slc16a family proteins have 12 transmembrane domains (Price et al. 1998). By skipping exon 2, the mature rmn mutant transcript lacks the initiator codon; however, the mRNA we isolated may still encode an N-terminally deleted Slc16a6a protein beginning with methionine codon 97 (Figs. 3D,E, 4C), although a Kozak sequence does not precede this codon. Likewise, leucine codon 86 (CUG) could serve as a cryptic site of alternative translation initiation. Antibodies raised against the extreme $\mathrm{C}$ terminus of Slc16a6a protein allowed us to unequivocally exclude these possibilities (Supplemental Fig. S5): Only wild-type Slc16a6a was detected by immunoblotting of proteins from dissected adult livers (Fig. 4D,E). The rmn mutation inactivates the slc16a6a gene.

\section{Slc16a6a is a ketone body transporter required in the liver during fasting}

The activity of Slc16a6 has not been functionally characterized in any organism, yet it is very likely that zebrafish Slc16a6a is an active MCT because it shows conservation of catalytic residues initially identified in rat Slc16al (Supplemental Fig S5). We expressed Slc16a6a heterologously in Xenopus oocytes and assessed whether the encoded protein can transport monocarboxylic acids, focusing on the most widely reported Slc16a family substrate (lactate) and two plausible substrates whose abundance increases in the fasting state: the major ketone body $\beta$-hydroxybutrate, and the branched-chain keto acid isovalerate, which is liberated from leucine catabolism and serves as a ketogenic precursor. Inward currents were generated in Slc16a6aexpressing oocytes perfused with $\beta$-hydroxybutyrate, but not with isovalerate or lactate (Fig. 4F). The current-tovoltage relationship indicates that, like all other previously studied Slc16a family members, Slc16a6a is not voltage-dependent (Fig. 4G). Thus, of the plausible substrates examined, Slc16a6a appears to be a selective $\beta$-hydroxybutyrate transporter. We were unable to detect currents in rmn mutant slc16aba-expressing oocytes (data not shown).

Since the liver is the major site of ketone body production in vertebrates (Phillips and Hird 1977), we
A

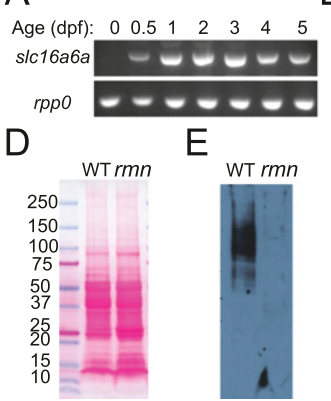

F
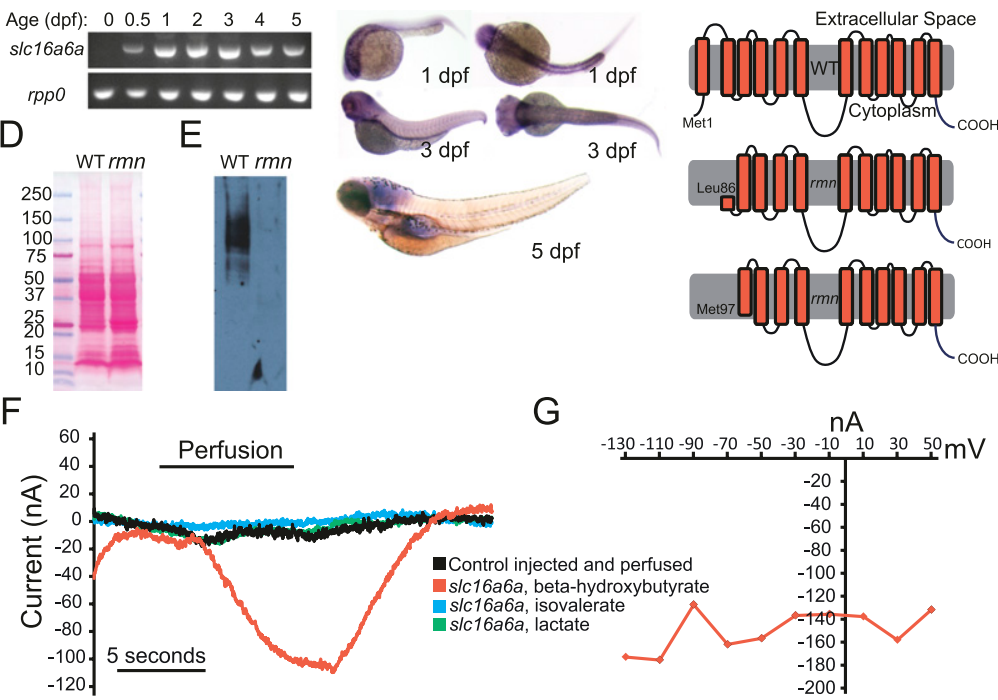

C

B
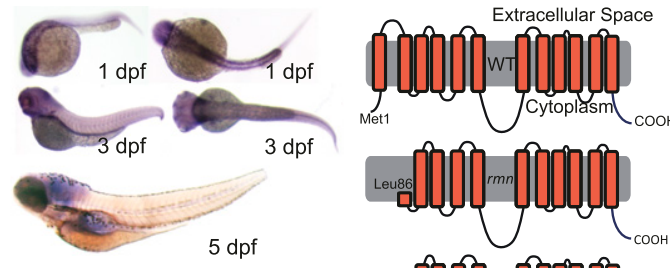

$\mathrm{COOH}_{\mathrm{COH}}$

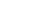

Figure 4. Slc16a6a is a $\beta$-hydroxybutyrate transporter. (A) RT-PCR of mature slc16a6a and rpp0 transcripts at the indicated ages. $(B)$ Whole-mount in situ hybridization with slc16a6a riboprobes. $(C)$ The topology of the predicted wild-type and hypothetical rmn Slc16a6a proteins. (D) Ponceau S staining of nitrocellulose membranes following transfer of proteins resolved by sodium dodecyl sulfate polyacrylamide electrophoresis. (E) Immunoblotting with custom anti-Slc16a6a IgGs of the membrane in $D$. The membrane was deliberately overexposed in order to increase the chance of detecting a smaller protein band in the rmn mutant. $(F)$ Current tracings of Xenopus oocytes expressing Slc16a6a (or control-injected oocytes, in black) that were perfused with monocarboxylic acids $(5 \mathrm{mM})$ for the indicated time period. Oocytes were voltageclamped to a holding potential of $-70 \mathrm{mV}$. $(G)$ Current-voltage relation of a Slc16a6a-expressing oocyte perfused with $\beta$-hydroxybutyrate. 
A

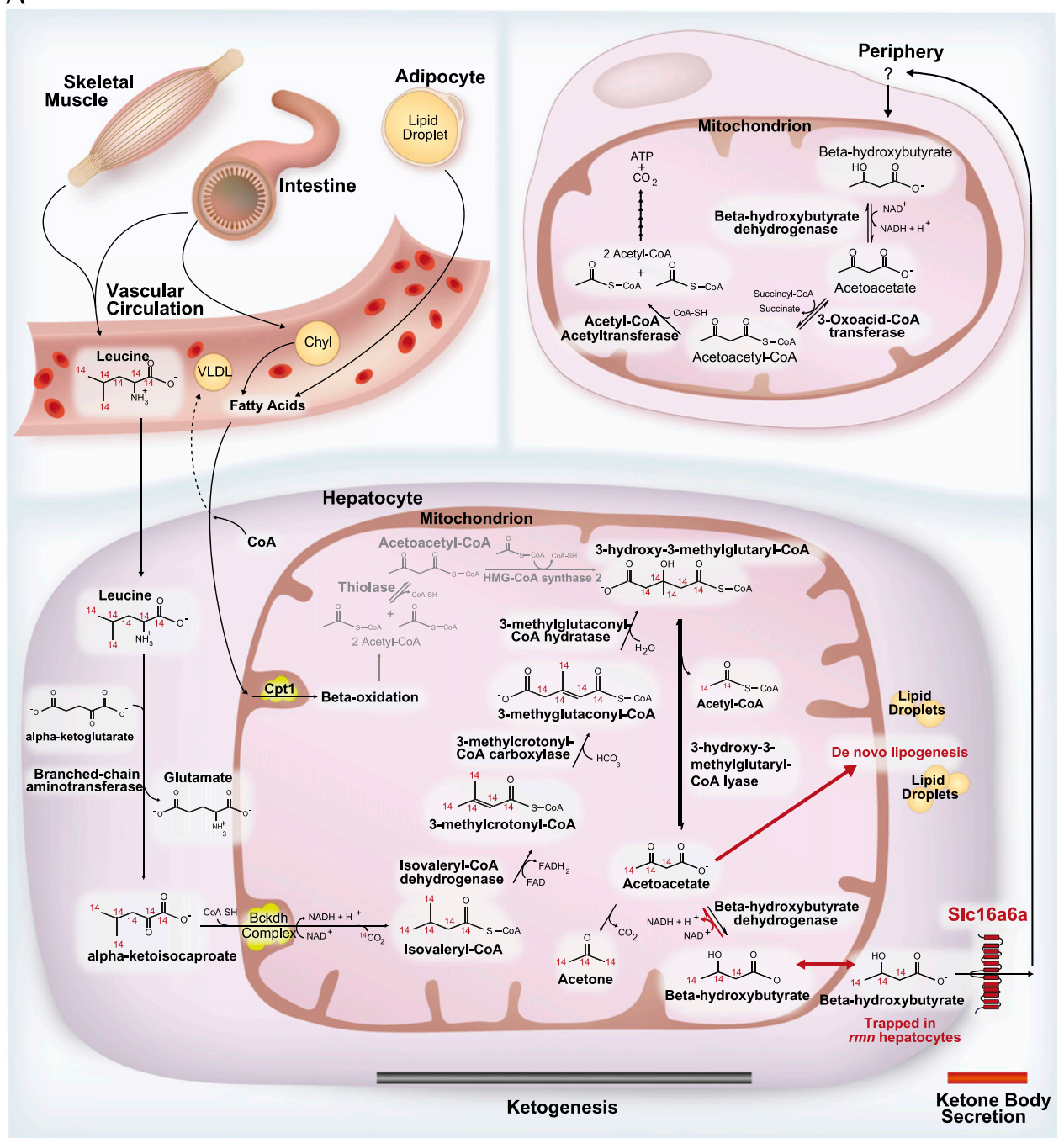

B

\begin{tabular}{|c|c|c|c|}
\hline Cholesterol & Cholesteryl e & ee Fatty $\mathrm{A}$ & Triacylglycero \\
\hline${ }^{14} \mathrm{C}$ incorporation ratio $0.83 \pm 0.04$ & $1.0 \pm 0.1$ & $1.3 \pm 0.1$ & $18+6$ \\
\hline
\end{tabular}

Figure 5. (A) A model of the role of Slc16a6a in hepatic ketone body metabolism. Dietary and stored reduced carbon atoms in the form of fatty acids (intestine and adipose, respectively) can be delivered to the liver, where, after partial $\beta$-oxidation, they can be repackaged as ketone bodies for feeding the peripheral organs during fasting. Triacylglycerol can also be packaged into VLDL particles (liver) or chylomicrons (intestine) for delivery to the periphery. The intestine and skeletal muscle also provide ketogenic amino acids, the most abundant of which is leucine. In birds, reptiles, and mammals, the rate-limiting enzyme of ketogenesis is mitochondrial HMG-CoA synthase 2 (gray reactions), which arose in birds through gene duplication of the cytosolic HMG-CoA synthase 1 gene (Boukaftane et al. 1994). Amphibians, fish, and other lower vertebrates have only the cytosolic, cholesterol-synthetic HMG-CoA synthase 1 and rely on increased flux through deamination and oxidation of leucine (which also occurs in higher vertebrates) to make mitochondrial HMGCoA for ketogenesis (Phillips and Hird 1977; Leblanc and Ballantyne 2000). Slc16a6a is a $\beta$-hydroxybutyrate-transporting MCT that is expressed in the liver. In rmn mutants, this transporter is inactivated, and the carbon atoms that would otherwise be secreted as fuel during fasting are diverted back to de novo lipogenesis and storage in lipid droplets. The peripheral ketone body transporters are not known ("?"), but Slc16a1, Slc16a7, and Slc16a3 are expressed in brain endothelial cells, neurons, and astrocytes, respectively (Pierre and Pellerin 2005). In zebrafish, there is evidence that Slc5a family members are expressed in muscle and can transport sodium hydroxybutyrate (Plata et al. 2007). (CoA) Coenzyme A; (Cpt1) Carnitine palmitoyl transferase 1; (Bckdh) Branched chain ketoacid dehydrogenase; (Chyl) chylomicron particle; (FAD) flavine adenine dinucleotide; $\left(\mathrm{FADH}_{2}\right)$ reduced $\mathrm{FAD}$; $\left(\mathrm{NAD}^{+}\right)$nicotinamide adenine dinucleotide; $\left(\mathrm{NADH}_{2}\right)$ reduced $\mathrm{NAD}$; (VLDL) very low-density lipoprotein particle. $(B)$ Larvae were incubated with $\mathrm{L}-\left[{ }^{14} \mathrm{C}(\mathrm{U})\right]$-leucine, and then lipids were analyzed as described in the Materials and Methods to calculate the ${ }^{14} \mathrm{C}$ incorporation ratio. Values greater than unity reflect increased relative incorporation of ketogenic precursor carbon atoms into neutral lipids in rmn mutants. 
hypothesize that the rmn mutation renders hepatocytes incapable of secreting ketone bodies (Fig. 5A). We tested this model by incubating wild-type and rmn mutant larvae in $\mathrm{L}-\left[{ }^{14} \mathrm{C}(\mathrm{U})\right]-$-leucine, a precursor of ketone bodies, and assessed for tracer incorporation into neutral lipids. A striking increase in relative incorporation of tracer into triacylglycerol, a modest increase into free fatty acids, and no increases into cholesterol and cholesteryl esters were seen in extracts of rmn mutants (Fig. 5B). This finding confirms our hypothesis that loss of hepatic ketone body transport via Slc16a6a causes diversion of carbon atoms into neutral storage lipids. This finding also suggests that the lack of increased serum ketone bodies in fasting wild-type animals may reflect very rapid clearance from the serum.

To formally assess whether Slc16a6a expression is required in the liver, we restored wild-type slc16a6a expression in rmn mutants using transgenic tools that allowed both broad and liver-specific transgene activation. The rmn phenotype can be rescued by transgenic expression of wild-type slc16a6a exclusively in the liver (Fig. 6A). This result indicates that while this MCT is broadly expressed (Fig. 4B), its major site of action in regulating hepatic lipid mass during fasting is the liver. We also found that forced expression of the human ortholog SLC16A6 in rmn mutant hepatocytes could rescue the rmn phenotype, underscoring that this evolutionarily conserved gene appears to function similarly in higher vertebrates.

Consistent with our hypothesis that the rmn mutation causes diversion of carbon atoms from ketone bodies to neutral lipids, we found changes in the abundance of select transcripts encoding enzymes and transcription factors involved in lipid and ketone body metabolism in adult livers under fed and fasted states (Table 1). In particular, there was induction of numerous Ppara targets, as expected in fasted animals (cpt1a [carnitine palmitoyl transferase $1 a]$ and the messages encoding proteins involved in $\beta$-oxidation). Interestingly, the ppara transcript was not altered, and we found that treatment with four different Ppara agonists did not rescue the rmn mutant phenotype, suggesting that this pathway is already maximally induced in rmn mutants (Supplemental Table S1). In addition, fasted adult $r m n$ mutant livers had increased abundance of transcripts encoding "adipogenic" transcription factors (srebf2 and cebpa), cholesterol biosynthetic enzymes, and enzymes of triacylglycerol synthesis. Taken together, this gene expression profile of adult livers suggests that disrupting hepatic ketone transport induces further expression of ketogeneic and branched-chain amino acid catabolic pathways. Also induced in an attempt to store carbon atoms that cannot be secreted are cholesterol biosynthetic and triacylglycerol biosynthetic genes. Likewise, fatty acyl oxidative pathways are up-regulated when ketone body transport is impaired.

As a final demonstration of the requirement of Slc16a6a in fasting physiology, we examined the consequences of feeding on the rmn phenotype. Gratifyingly, rmn mutants were not steatotic when fed from 5 to $12 \mathrm{dpf}$ (Fig. 6B); however, withdrawal of food from rmn mutants, but not from wild-type animals, led to hepatic steatosis

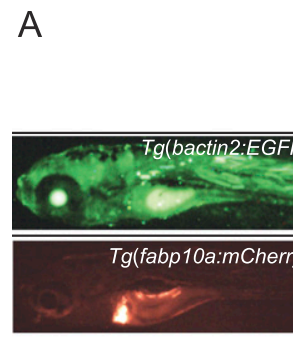

B

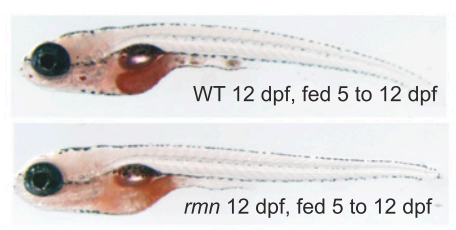

D

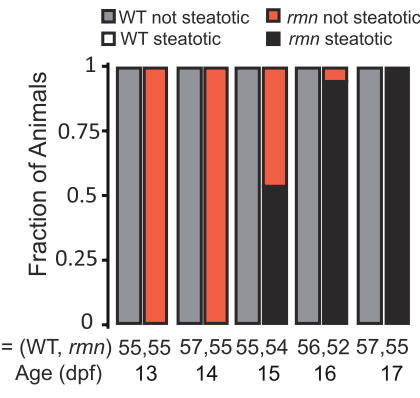

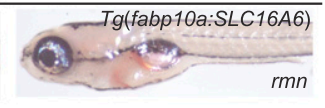

C

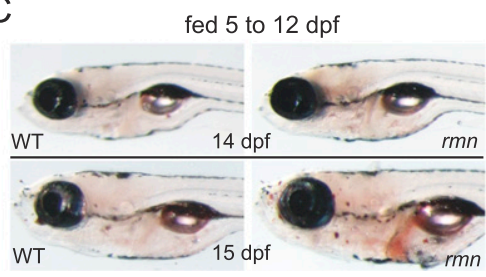

E

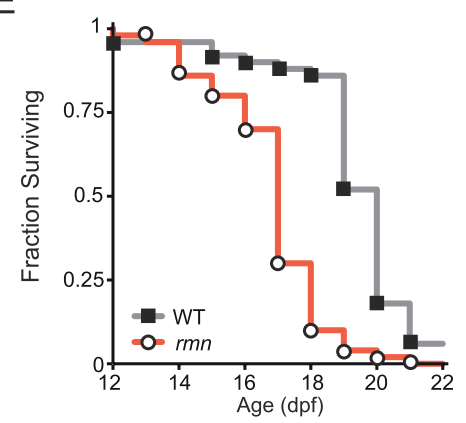

Figure 6. Genetic and nutritional rescue of $r m n \mathrm{mu}-$ tants. (A) Single-cell, homozygous rmn mutant embryo larvae were injected with wild-type zebrafish slc16a6a or human SLC16A6 cDNAs under the control of either bactin2 (broadly expressed) or fabp10a (liver-limited) promoter elements. Larvae were then stained with ORO on 6 dpf. $(B)$ Whole-mount ORO staining of larvae fed from 5 to $12 \mathrm{dpf}$. The intestinal lumen contents were also stained with ORO, reflecting the lipid content of the larval diet. $(C)$ Whole-mount ORO staining of larvae fed from 5 to $12 \mathrm{dpf}$ and then removed from food. (D) In previously fed rmn mutants, hepatic steatosis appears after $3 \mathrm{~d}$ of fasting. (E) Survival curve for animals fed from 5 to $12 \mathrm{dpf}$ and then removed from food ( $n=50$ larvae of each genotype). The median age at death was $20 \pm 2 \mathrm{dpf}$ for wild-type larvae and $18 \pm 1.7$ dpf for $r m n$ mutant larvae (log rank test: $\chi^{2}=62.3$ on one degree of freedom; $P=2.9 \times 10^{-16}$ ). 
Table 1. Quantitative RT-PCR gene expression profiling

\begin{tabular}{|c|c|c|c|c|c|}
\hline \multirow[b]{2}{*}{ Gene } & \multirow[b]{2}{*}{ Protein } & \multicolumn{2}{|c|}{ Wild type } & \multicolumn{2}{|c|}{ rmn mutant } \\
\hline & & Fasted & Fed & Fasted & Fed \\
\hline \multicolumn{6}{|c|}{ Transcription factors regulating lipid metabolism } \\
\hline cebpa & CCAAT/enhancer-binding protein $\alpha$ & $1.0 \pm 0.01$ & $1.8 \pm 0.01^{\mathrm{a}}$ & $2.6 \pm 0.03^{\mathrm{a}}$ & $7.2 \pm 0.03^{\mathrm{a}, \mathrm{b}}$ \\
\hline pparab & Peroxisome proliferator-activated receptor $\alpha \mathrm{b}$ & $1.0 \pm 0.02$ & $0.8 \pm 0.02$ & $0.9 \pm 0.02$ & $1.0 \pm 0.03$ \\
\hline pparg & Peroxisome proliferator-activated receptor $\gamma$ & $1.0 \pm 0.004$ & $1.0 \pm 0.007$ & $1.1 \pm 0.01$ & $2.7 \pm 0.02^{\mathrm{a}}$ \\
\hline nr1h3 & Liver $\mathrm{x}$ receptor $\alpha$ & $1.0 \pm 0.05$ & $0.7 \pm 0.01$ & $0.8 \pm 0.01$ & $0.8 \pm 0.01$ \\
\hline srebf1 & Sterol regulatory element-binding transcription factor 1 & $1.0 \pm 0.03$ & $1.5 \pm 0.05$ & $1.2 \pm 0.04$ & $0.9 \pm 0.03$ \\
\hline srebf2 & Sterol regulatory element-binding transcription factor 2 & $1.0 \pm 0.1$ & $3.0 \pm 0.1^{\mathrm{a}}$ & $2.1 \pm 0.01^{\mathrm{a}}$ & $6.6 \pm 0.4^{\mathrm{a}, \mathrm{b}}$ \\
\hline mlxip & MLX-interacting protein (Chrebp) & $1.0 \pm 0.03$ & $1.3 \pm 0.03$ & $1.7 \pm 0.05$ & $0.6 \pm 0.03$ \\
\hline \multicolumn{6}{|c|}{ Cholesterol synthesis } \\
\hline hmgcra & 3-Hydroxy-3-methylglutaryl-coenzyme A reductase a & $1.0 \pm 0.03$ & $25.3 \pm 1.3^{\mathrm{a}}$ & $63 \pm 1^{\mathrm{a}}$ & $3050 \pm 30^{\mathrm{a}, \mathrm{b}}$ \\
\hline hmgcs1 & 3-Hydroxy-3-methylglutaryl-coenzyme A synthase 1 & $1.0 \pm 0.01$ & $41.3 \pm 0.6^{\mathrm{a}}$ & $5.9 \pm 0.1^{\mathrm{a}}$ & $437 \pm 6^{\mathrm{a}, \mathrm{b}}$ \\
\hline$h m g c l$ & 3-Hydroxy-3-methylglutaryl-coenzyme A lyase & $1.0 \pm 0.06$ & $1.0 \pm 0.06$ & $1.7 \pm 0.1^{\mathrm{a}, \mathrm{b}}$ & $1.0 \pm 0.06$ \\
\hline \multicolumn{6}{|c|}{ Branched-chain ketoacid and ketone body metabolism } \\
\hline bcat2 & Branched-chain aminotransferase, mitochondrial & $1.0 \pm 0.02$ & $4.14 \pm 0.06^{\mathrm{a}}$ & $8.1 \pm 0.1^{\mathrm{a}, \mathrm{b}}$ & $2.27 \pm 0.03^{\mathrm{a}}$ \\
\hline$b c k d h b l$ & Branched-chain ketoacid dehydrogenase subunit $b$, like & $1.0 \pm 0.02$ & $1.92 \pm 0.03^{\mathrm{a}}$ & $2.84 \pm 0.04^{\mathrm{a}, \mathrm{b}}$ & $1.34 \pm 0.02^{\mathrm{a}}$ \\
\hline bdh1 & 3-Hydroxybutyrate dehydrogenase, mitochondrial & $1.0 \pm 0.06$ & $1.0 \pm 0.06$ & $2.5 \pm 0.14^{\mathrm{a}, \mathrm{b}}$ & $0.5 \pm 0.03^{\mathrm{a}}$ \\
\hline oxct1b & 3-Oxoacid coenzyme A transferase $1 \mathrm{~b}$ (liver-specific) & $1.0 \pm 0.02$ & $4.4 \pm 0.1^{\mathrm{a}}$ & $8.5 \pm 0.2^{\mathrm{a}, \mathrm{b}}$ & $10.6 \pm 0.4^{\mathrm{a}}$ \\
\hline \multicolumn{6}{|c|}{$\begin{array}{l}\text { De novo fatty acid and triacylglycerol synthesis and } \\
\text { VLDL secretion }\end{array}$} \\
\hline acc1 & Acetyl-coenzyme A carboxylase 1 & $1.0 \pm 0.07$ & $51.9 \pm 1.3^{\mathrm{a}}$ & $0.7 \pm 0.01$ & $16.8 \pm 0.2^{\mathrm{a}}$ \\
\hline fasn & Fatty acid synthase & $1.0 \pm 0.05$ & $70.5 \pm 1.0^{\mathrm{a}}$ & $1.2 \pm 0.04$ & $28.0 \pm 0.4^{\mathrm{a}}$ \\
\hline scd & Steroyl-CoA desaturase ( $\Delta$-9-desaturase) & $1.0 \pm 0.02$ & $8,520 \pm 90^{\mathrm{a}}$ & $58 \pm 1^{\mathrm{a}}$ & $5080 \pm 30^{\mathrm{a}}$ \\
\hline elov15 & Elongation of long chain fatty acids family member 5 & $1.0 \pm 0.15$ & $1.0 \pm 0.23$ & $0.3 \pm 0.04^{\mathrm{a}, \mathrm{b}}$ & $0.3 \pm 0.03^{\mathrm{a}, \mathrm{b}}$ \\
\hline fads2 & Fatty acid desaturase 2 ( $\Delta$-5- and $\Delta$-6-desaturase) & $1.0 \pm 0.01$ & $1.58 \pm 0.01$ & $0.4 \pm 0.002$ & $0.7 \pm 0.004$ \\
\hline agpat4 & 1-Acylglycerol-3-phosphate $O$-acyltransferase 4 & $1.0 \pm 0.02$ & $5.0 \pm 0.1^{\mathrm{a}}$ & $16.8 \pm 0.4^{\mathrm{a}, \mathrm{b}}$ & $9.9 \pm 0.3^{\mathrm{a}, \mathrm{b}}$ \\
\hline ppap $2 a b$ & Phosphatidic acid phosphatase $2 \mathrm{a} b$ & $1.0 \pm 0.03$ & $2.8 \pm 0.1$ & $1.2 \pm 0.03$ & $0.7 \pm 0.02$ \\
\hline dgat2 & Diacylglycerol O-acyltransferase 2 & $1.0 \pm 0.1$ & $0.9 \pm 0.1$ & $14.9 \pm 1.9^{\mathrm{a}, \mathrm{b}}$ & $22.6 \pm 2.3^{\mathrm{a}, \mathrm{b}}$ \\
\hline$m t p$ & Microsomal triglyceride transfer protein & $1.0 \pm 0.17$ & $2.2 \pm 0.4^{\mathrm{a}}$ & $1.7 \pm 0.3^{\mathrm{a}}$ & $3.4 \pm 0.6^{\mathrm{a}}$ \\
\hline \multicolumn{6}{|c|}{ Fatty acid oxidation } \\
\hline cpt1a & Carnitine O-palmitoyltransferase I (liver isoform) & $1.0 \pm 0.03$ & $1.3 \pm 0.04^{\mathrm{a}}$ & $1.4 \pm 0.04^{\mathrm{a}}$ & $1.2 \pm 0.04^{\mathrm{a}}$ \\
\hline acadl & Acyl-coenzyme A dehydrogenase, long chain & $1.0 \pm 0.03$ & $5.1 \pm 0.1^{\mathrm{a}}$ & $3.2 \pm 0.04^{\mathrm{a}}$ & $3.8 \pm 0.04^{\mathrm{a}}$ \\
\hline acadm & $\begin{array}{l}\text { Acyl-coenzyme A dehydrogenase, C- } 4 \text { to C-12 } \\
\text { straight chain }\end{array}$ & $1.0 \pm 0.1$ & $2.7 \pm 0.04^{\mathrm{a}}$ & $2.7 \pm 0.04^{\mathrm{a}}$ & $2.3 \pm 0.2^{\mathrm{a}}$ \\
\hline ehhadh & $\begin{array}{l}\text { Enoyl-CoA, hydratase/3-hydroxyacyl CoA } \\
\text { dehydrogenase }\end{array}$ & $1.0 \pm 0.03$ & $2.6 \pm 0.08^{\mathrm{a}}$ & $1.6 \pm 0.05^{\mathrm{a}}$ & $2.3 \pm 0.08^{\mathrm{a}}$ \\
\hline$m t-n d 1$ & NADH dehydrogenase 1 , mitochondrial & $1.0 \pm 0.1$ & $2.1 \pm 0.1^{\mathrm{a}}$ & $2.6 \pm 0.03^{\mathrm{a}}$ & $2.0 \pm 0.05^{\mathrm{a}}$ \\
\hline$m t-c o 1$ & Cytochrome c oxidase 1 , mitochondrial & $1.0 \pm 0.02$ & $1.3 \pm 0.01^{\mathrm{a}}$ & $1.3 \pm 0.02^{\mathrm{a}}$ & $1.4 \pm 0.01^{\mathrm{a}}$ \\
\hline$m t-a t p 6$ & ATP synthase 6 , mitochondrial & $1.0 \pm 0.1$ & $0.9 \pm 0.03$ & $0.8 \pm 0.02$ & $1.2 \pm 0.03$ \\
\hline \multicolumn{6}{|c|}{ Slc16a6 paralogs } \\
\hline slc16aba & Solute carrier family $16 a$, member $6 a$ & $1.0 \pm 0.02$ & $0.8 \pm 0.02$ & $1.2 \pm 0.02$ & $0.1 \pm 0 . .002$ \\
\hline Slc16a6b & Solute carrier family $16 \mathrm{a}$, member $6 \mathrm{~b}$ & $1.0 \pm 0.02$ & $2.0 \pm 0.04$ & $2.6 \pm 0.05$ & $0.4 \pm 0.01^{\mathrm{a}, \mathrm{b}}$ \\
\hline
\end{tabular}

${ }^{\mathrm{a}} P<0.01$ versus wild type fasted.

${ }^{\mathrm{b}} P<0.01$ versus wild type fed.

(Fig. 6C,D). To assess whether the development of steatosis in previously fed animals affected survival, wild-type and $r m n$ mutant larvae were fed from 5 to $12 \mathrm{dpf}$ and then scored for death after food was withdrawn. Again, as expected, wild-type animals survived longer than rmn mutant animals (Fig. 6E). Thus, rmn mutant larvae develop hepatic steatosis under nutrient-deficient states and are rendered sensitive to starvation.

\section{Discussion}

In diverse metazoan species from flies (Gutierrez et al. 2007) to humans (Gibbons et al. 2000), fasting causes mobilization of energy substrates from the peripheral organs in the form of fatty acids from adipose and amino acids from muscle. Upon return to the liver, these metabolites are partially oxidized into ketone bodies, a fuel source that can be used by the brain. These molecules and the glucose liberated from hepatic glycogenolysis and gluconeogenesis are used as fuel in peripheral organs during fasting (Cahill 2006). The availability of ketone bodies for use by organs other than the liver reflects the lower hepatic abundance of 3-oxoacid coenzyme A (CoA) transferase 1, the enzyme required to break down ketone bodies (Laffel 1999).

In inbred strains of mice, the influx of fatty acids from adipose tissue during fasting leads to their re-esterification and temporary storage in the liver. The extent of this steatosis varies based on genetic background and reflects differences in muscle fat oxidation and the degree of 
adipose lipolysis (Lin et al. 2005; Guan et al. 2009). Furthermore, there is an inverse association between hepatic lipid levels and serum ketone body concentrations in fasted mice (Lin et al. 2005). Normal humans subjected to fasting show an increase in hepatic lipid content in response to a $36-\mathrm{h}$ fast, but there is variation in the extent of lipid accumulation (Moller et al. 2008).

Here we described the use of a genetic screen to isolate novel regulators of lipid metabolism. Our approach led to the identification of a nutritionally suppressible hepatic steatosis mutant, rmn. In contrast to previously reported, lethal mutants, the steatosis seen in rmn mutants is neither preceded nor followed by the development of degenerative changes in hepatocyte architecture or fulminant inflammation. The rmn mutation causes a complete loss of Slc16a6a expression, as revealed by the absence of mutant protein expression. The mutated gene encodes a MCT that transports $\beta$-hydroxybutyrate Slc16a6a. Mutation of this ketone body transporter triggers accumulation of neutral lipids in fasted livers and renders animals sensitive to starvation. The rmn mutant phenotype can be rescued by forced expression of wildtype zebrafish slc16a6a exclusively in the liver. Expression of the human ortholog SLC16A6 in the liver also rescues the mutant phenotype. Taken together, our lossof-function genetic data, transgenic rescue observations, transporter characterization, radiotracer study, and nutritional manipulations indicate that Slc16a6a is a hepatic ketone body transporter required during fasting (Fig. 5).

Placed in a broader context, the rmn mutant phenotype is reminiscent of that seen when Ppara function is lost. In Ppara $^{-/-}$mice, there is fasting hepatic steatosis and hypoketonemia (Kersten et al. 1999; Hashimoto et al. 2000). Liver-restricted inactivation of Tbl1, which encodes Transducin $\beta$-like 1 , a heterodimerization partner of Ppara, causes phenotypes similar to Ppara inactivation (Kulozik et al. 2011). Several Ppara targets are induced in rmn mutants beyond the anticipated increases seen in fasted wild-type animals (Table 1). This induction is probably maximal because treatment with synthetic Ppara ligands did not rescue the rmn mutant phenotype (Supplemental Table S1). This lack of pharmacological rescue contrasts with the complete amelioration of fasting hepatic steatosis by administration of a single dose of synthetic Ppara ligand to mice bearing liver-specific inactivation of Fasn, the gene encoding Fatty acid synthase, whose product catalyzes the production of precursors for endogenous Ppara ligands (Chakravarthy et al. 2005).

The rmn phenotype is also reminiscent of the phenotype of mice with liver-specific deletion of Pnpla2, in which there is loss of hepatic expression of Adipose triglyceride lipase. These mice develop steatohepatitis, marked by a 99\% reduction of Ppara transcript levels and a 99\% reduction in expression of the central Ppara target transcript Cpt1a (Wu et al. 2011). Similar to rmn mutant larvae, Srebf1 and Dgat 2 transcripts were appropriately reduced in this model; however, the fasting serum $\beta$-hydroxybutyrate was not altered. Thus, nutrient trapping through impaired hepatic lipolysis has some but not all of the hallmarks of impaired hepatic ketone body secretion.
In another mouse model with impaired Ppara function, there are further subtle differences in fasting phenotypes. Genetic activation of the Target of rapamycin (Tor) signaling pathway by liver-specific deletion of the Tor antagonist Tsc1 (LiTsc1KO) causes impaired ketogenesis in fasting adult mice. Furthermore, Tor-dependent suppression of ketogenesis occurs through suppression of Ppara-induced gene expression. Puzzlingly, LiTsc1KO mice do not develop fasting hepatic steatosis, despite impaired induction of Ppara target genes (Sengupta et al. 2010). Thus, alterations in ketone body production can be divorced from hepatic lipid accumulation, even when Ppara signaling is impaired.

Since intrahepatic lipid accumulation varies in healthy human subjects (Moller et al. 2008) and among inbred strains of mice (Lin et al. 2005), it would be informative to assess whether there is an inverse association of fasting ketone levels and intrahepatic lipid accumulation in normal humans subjected to prolonged fasting. Whether altered fasting liver metabolism influences NAFLD development is also an open question. Likewise, because $\beta$-hydroxybutyrate is both a major nutrient and a signaling molecule that coordinates fasting metabolism (Laeger et al. 2010), pharmacologic manipulation of ketone body transport in such states as fasting, uncontrolled type 1 diabetes mellitus, dyslipidemia, and obesity holds therapeutic promise (Veech 2004).

\section{Materials and methods}

\section{Fish}

The $r m n^{s 951}$ allele is carried on a [Tg(ins:dsRed ${ }^{m 1081}$; $\mathrm{Tg}(\mathrm{fabp} 10 \mathrm{a}$ : dsRed $)^{g z 4}$; Tg(ela31:GFP $\left.)^{g^{2}}\right] \times$ WIK background. Outcrosses to TL or unrelated $\mathrm{AB}$ had no effect on the penetrance or expressivity of the mutant phenotype. These studies were approved by the University of Utah Institutional Animal Care and Use Committee and the Radiation Safety Committee.

\section{ORO staining and screening}

Forty to 50 6-dpf animals from individual F2 in-crosses were fixed and stained as described previously (Schlegel and Stainier 2006). Only fully penetrant ( $25 \%$ of the animals) and fully expressive (unambiguous, uniform increase in liver staining) mutants were analyzed further.

\section{Transgenic rescue constructs}

Tol2 transposase-mediated transgenic expression of wild-type slc16a6a cDNA was achieved by placing the slc16a6a cDNA under the control of either a $5.3-\mathrm{kb}$ bactin2 promoter (for broad expression) or a $2.8-\mathrm{kb}$ fabp10a promoter (for liver-specific expression) in a Tol2 transposase destination vector that was coinjected with Tol2 mRNA into single-cell, fertilized embryos (Her et al. 2003; Kwan et al. 2007). Parallel constructs driving EGFP under the bactin2 promoter and mCherry under the fabp10a promoter were injected to confirm that broad or liverlimited expression was achieved (respectively) with our experimental design. The human SLC16A6 cDNA was placed under control of the fabp10a promoter.

\section{Electrophysiology}

Electrophysiological characterization of Slc16a6a was performed at Ecocyte Biosciences using a Robocyte automated two-elec- 
trode voltage clamp recorder (Schnitzler et al. 2003). Xenopus oocytes were harvested and injected with either empty pGEMT vector or pGEMT containing slc16a6a cDNA preceded by a Kozak translation initiation sequence (5'-GCCACCACC-3'). The cDNA was cloned in the T7 polymerase reading direction. Four days after injection, oocytes were clamped to a holding potential of $-70 \mathrm{mV}$, and currents were recorded at a sampling rate of $1000 \mathrm{~Hz}$ at room temperature. Substrates $15 \mathrm{mM}$ $\beta$-hydroxybutyrate, lactate, or isovalerate) were applied for 10 $\mathrm{sec}$, and the inward current was evaluated.

\section{Metabolite analyses}

Following thin-layer chromatographic resolution of individual species, lipids were visualized by placing the silica plates in a sealed chamber containing iodide crystals (Schlegel and Stainier 2006). The resolved lipids were scraped off the plates and analyzed individually. Cholesteryl oleate, glyceryl triolein, oleic acid, and cholesterol standards were from Sigma. The perchlorate method (Snyder and Stephens 1959) was used for quantifying triacylglycerol exactly as we described previously (Schlegel and Stainier 2006). Cholesterol and cholesteryl esters were quantified using the $o$-phthalaldehyde method (Rudel and Morris 1973). Free fatty acids were determined using a commercially available (BioVision) assay involving enzymatic activation of coenzyme A derivatives, followed by oxidation in the presence of $N$-ethylmaleimide (Mizuno et al. 1980). $\beta$-Hydroxybutyrate was detected enzymatically (Caymen Chemicals). Capillary glucose was measured with a Roche glucometer. Serum was diluted in phosphatebuffered saline (PBS) with $1 \mathrm{mM}$ EDTA prior to lipid extraction and thin-layer chromatographic analysis of triacylglycerol.

\section{Radiotracer study}

Larvae (5 dpf) were incubated with $10 \mathrm{mM} \mathrm{L-}\left[{ }^{14} \mathrm{C}(\mathrm{U})\right]$-leucine tracer for $72 \mathrm{~h}$, and then total lipids were extracted, separated by thin-layer chromatography, and detected by autoradiography using a Molecular Dynamics PhosphorImager. Band intensities (signal) were quantified with GelQuantNET. Band intensities were normalized to the masses of lipid present in the wild-type and rmn samples, and a ratio of incorporation relative to mass was calculated as follows: $\left({ }^{14} \mathrm{C} \text { signal } / \text { mass lipid }\right)_{r m n} /\left({ }^{14} \mathrm{C} \text { signal } / \text { mass lipid }\right)_{\mathrm{WT}}$.

\section{Ppara agonist treatment}

Forty 5-dpf larvae were treated with clofibrate, fenofibrate, gemfibrozil, or WY 14,643 for $24 \mathrm{~h}$. Animals were then fixed and stained with ORO and scored for the presence of hepatic steatosis.

\section{In situ hybridization}

A 461-bp riboprobe was prepared from the $3^{\prime}$ untranslated region (UTR) and final two exons of slc16a6a using the following primers to amplify the sequence: forward, 5'-GCAGAAGGAGGAAAAA TGGA-3'; and reverse, 5'-GGAAGAATGGTTAACCCAAA-3'. The product was cloned into pGEMT, and an antisense probe was transcribed using T7 RNA polymerase (Promega) and DIG-labeled nucleotides (Roche). Larvae were digested with proteinase K prior to hybridization exactly as described (Thisse and Thisse 2008). Signal was detected with a DIG detection kit (Roche).

\section{Immunoblotting}

Six wild-type and $r m n$ mutant livers were sonicated in $300 \mu \mathrm{L}$ of 50 $\mathrm{mM}$ Tris (pH 8.0), $150 \mathrm{mM} \mathrm{NaCl}, 1.0 \%$ IGEPAL CA-630, 0.25\% sodium deoxycholate, $0.1 \%$ SDS, and $1 \mathrm{mM}$ EDTA supplemented with protease and phosphatase inhibitor cocktails (Roche Complete MINI and PhosSTOP). Debris was pelleted by centrifugation. Twenty micrograms of protein (concentration determined with a BCA kit, Thermo) from each lysate was separated by SDS-PAGE, transferred to nitrocellulose membranes, and detected with antizebrafish Slc16a6a (21st Century Biochemicals).

\section{Histology}

Hematoxylin and eosin sectioning of adult livers was performed by AML Laboratories.

\section{Confocal microscopy}

Larvae (6 dpf) were fixed in PBS containing 4\% formaldehyde, washed in PBS containing $0.1 \%$ saponin (S-PBS), incubated with S-PBS containing BODIPY 493/503 (10 mg/L), washed with S-PBS, and mounted in glycerol after partial dissection of the liver from the body. Confocal images were taken using a Zeiss 510 confocal microscope.

\section{Electron microscopy}

Electron microscopy was performed at the University of Utah Electron Microscopy Facility and the J. David Gladstone Institute's Electron Microscopy Core Laboratory. Larvae (6 dpf) were fixed in $2.5 \%$ paraformaldehyde and $1 \%$ glutaraldehyde in $100 \mathrm{mM}$ sodium cacodylate ( $\mathrm{pH}$ 7.4), counterstained with $\mathrm{OsO}_{4}$, and sectioned at the level of the liver.

\section{Statistical analysis}

The two-sided Student's $t$-test was used to compare relative gene expression in fasted and fed animals and to compare lipid and protein levels in whole-larval extracts and dissected adult livers. For survival analysis, the log rank test was performed using freeware (http://bioinf.wehi.edu.au/software/russell/logrank).

Other methods are described in Supplemental Material.

\section{Acknowledgments}

We thank Carl S. Thummel, Don L. Gibbons, E. Dale Abel, Elizabeth A. Leibold, Jared Rutter, Ivana De Domenico, Jerry Kaplan, Philipp Gut, and David J. Grunwald for comments; Takuya Sakaguchi, Leonard Lipovich, and H. Joseph Yost for advice on positional cloning; Timothy E. Graham for use of his RT-PCR instrument; and Diana Lim for improving Figure 5A. This work was supported by the U.S. National Institutes of Health Grant K08-DK078605, a UCSF Diabetes Education and Research Pilot and Feasibility Award (P30-DK063720), and funds from the University of Utah Molecular Medicine (U2M2) Program to A.S. R.M.A. was supported by a Juvenile Diabetes Research Foundation fellowship. D.Y.R.S. was supported by grants from the U.S. National Institutes of Health (R01-DK060322) and the Packard Foundation.

\section{References}

Akimitsu M, Shin-ichi H, Daisuke K, Takanori N, Tomoko J, Davin HES, Satoshi O, Nobuaki O, Kenta H, Sayaka K, et al. 2008. Mutation in the abcb7 gene causes abnormal iron and fatty acid metabolism in developing medaka fish. Dev Growth Differ 50: 703-716. 
Anderson RM, Bosch JA, Goll MG, Hesselson D, Dong PD, Shin D, Chi NC, Shin CH, Schlegel A, Halpern M et al. 2009. Loss of Dnmtl catalytic acitivity reveals multiple roles for DNA methylation during pancreas development and regeneration. Dev Biol 334: 213-223.

Babin PJ, Vernier JM. 1989. Plasma lipoproteins in fish. J Lipid Res 30: 467-489.

Bonen A, Heynen M, Hatta H. 2006. Distribution of monocarboxylate transporters MCT1-MCT8 in rat tissues and human skeletal muscle. Appl Physiol Nutr Metab 31: 31-39.

Boukaftane Y, Duncan A, Wang S, Labuda D, Robert M-F, Sarrazin J, Schappert K, Mitchell GA. 1994. Human mitochondrial HMG CoA synthase: Liver cDNA and partial genomic cloning, chromosome mapping to 1p12-p13, and possible role in vertebrate evolution. Genomics 23: 552559.

Browning JD, Horton JD. 2004. Molecular mediators of hepatic steatosis and liver injury. J Clin Invest 114: 147-152.

Cahill GF. 2006. Fuel metabolism in starvation. Annu Rev Nutr 26: $1-22$.

Chakravarthy MV, Pan Z, Zhu Y, Tordjman K, Schneider JG, Coleman T, Turk J, Semenkovich CF. 2005. 'New' hepatic fat activates PPAR $\alpha$ to maintain glucose, lipid, and cholesterol homeostasis. Cell Metab 1: 309-322.

Clark JM. 2006. The epidemiology of nonalcoholic fatty liver disease in adults. I Clin Gastroenterol 40: S5-S10. doi: 10.1097/01.mcg.0000168638.84840.ff.

Cohen JC, Horton JD, Hobbs HH. 2011. Human fatty liver disease: Old questions and new insights. Science 332: 1519-1523.

Gibbons GF, Islam K, Pease RJ. 2000. Mobilisation of triacylglycerol stores. Biochim Biophys Acta 1483: 37-57.

Goodwin TJD, Poulter RT. 2004. A new group of tyrosine recombinase-encoding retrotransposons. Mol Biol Evol 21: 746-759.

Guan H-P, Goldstein JL, Brown MS, Liang G. 2009. Accelerated fatty acid oxidation in muscle averts fasting-induced hepatic steatosis in SJL/J mice. J Biol Chem 284: 24644-24652.

Gutierrez E, Wiggins D, Fielding B, Gould AP. 2007. Specialized hepatocyte-like cells regulate Drosophila lipid metabolism. Nature 445: 275-280.

Hashimoto T, Cook WS, Qi C, Yeldandi AV, Reddy JK, Rao MS. 2000. Defect in peroxisome proliferator-activated receptor $\alpha$-inducible fatty acid oxidation determines the severity of hepatic steatosis in response to fasting. I Biol Chem 275: 28918-28928.

Her GM, Chiang C-C, Chen W-Y, Wu J-L. 2003. In vivo studies of liver-type fatty acid binding protein (L-FABP) gene expression in liver of transgenic zebrafish (Danio rerio). FEBS Lett 538: $125-133$.

Hooper AJ, Adams LA, Burnett JR. 2011. Genetic determinants of hepatic steatosis in man. J Lipid Res 52: 593-617.

Ibabe A, Grabenbauer M, Baumgart E, Fahimi D, Cajaraville M. 2002. Expression of peroxisome proliferator-activated receptors in zebrafish. Histochem Cell Biol 118: 231-239.

Kersten S, Seydoux J, Peters JM, Gonzalez FJ, Desvergne B, Wahli W. 1999. Peroxisome proliferator-activated receptor $\alpha$ mediates the adaptive response to fasting. J Clin Invest 103: 1489-1498.

Kulozik P, Jones A, Mattijssen F, Rose Adam J, Reimann A, Strzoda D, Kleinsorg S, Raupp C, Kleinschmidt J, MüllerDecker K, et al. 2011. Hepatic deficiency in transcriptional cofactor TBL1 promotes liver steatosis and hypertriglyceridemia. Cell Metab 13: 389-400.

Kwan KM, Fujimoto E, Grabher C, Mangum BD, Hardy ME, Campbell DS, Parant JM, Yost HJ, Kanki JP, Chien C-B. 2007. The Tol2kit: A multisite gateway-based construction kit for
Tol2 transposon transgenesis constructs. Dev Dyn 236: 3088-3099.

Laeger T, Metges CC, Kuhla B. 2010. Role of $\beta$-hydroxybutyric acid in the central regulation of energy balance. Appetite 54: 450-455.

Laffel L. 1999. Ketone bodies: A review of physiology, pathophysiology and application of monitoring to diabetes. Diabetes Metab Res Rev 15: 412-426.

Leblanc PJ, Ballantyne JS. 2000. Novel aspects of the activities and subcellular distribution of enzymes of ketone body metabolism in the liver and kidney of the goldfish, Carassius auratus. J Exp Zool 286: 434-439.

Lin X, Yue P, Chen Z, Schonfeld G. 2005. Hepatic triglyceride contents are genetically determined in mice: Results of a strain survey. Am I Physiol Gastrointest Liver Physiol 288: G1179-G1189. doi: 10.1152/ajpgi.00411.2004.

Liou I, Kowdley KV. 2006. Natural history of nonalcoholic steatohepatitis. I Clin Gastroenterol 40: S11-S16. doi: 10.1097/01.mcg.0000168644.23697.31.

Matthews RP, Lorent K, Manoral-Mobias R, Huang Y, Gong W, Murray IVI, Blair IA, Pack M. 2009. TNF $\alpha$-dependent hepatic steatosis and liver degeneration caused by mutation of zebrafish $s$-adenosylhomocysteine hydrolase. Development 136: $865-875$.

Mizuno K, Toyosato M, Yabumoto S, Tanimizu I, Hirakawa H. 1980. A new enzymatic method for colorimetric determination of free fatty acids. Anal Biochem 108: 6-10.

Moller L, Stodkilde-Jorgensen H, Jensen FT, Jorgensen JOL. 2008. Fasting in healthy subjects is associated with intrahepatic accumulation of lipids as assessed by $1 \mathrm{H}$-magnetic resonance spectroscopy. Clin Sci (Lond) 114: 547-552.

Nugent C, Younossi ZM. 2007. Evaluation and management of obesity-related nonalcoholic fatty liver disease. Nat Clin Pract Gastroenterol Hepatol 4: 432-441.

Phillips JW, Hird FJ. 1977. Ketogenesis in vertebrate livers. Comp Biochem Physiol B 57: 133-138.

Pierre K, Pellerin L. 2005. Monocarboxylate transporters in the central nervous system: Distribution, regulation and function. J Neurochem 94: 1-14.

Plata C, Sussman CR, Sindić A, Liang JO, Mount DB, Josephs ZM, Chang M-H, Romero MF. 2007. Zebrafish Slc5a12 encodes an electroneutral sodium monocarboxylate transporter (SMCTn). J Biol Chem 282: 11996-12009.

Price NT, Jackson VN, Halestrap AP. 1998. Cloning and sequencing of four new mammalian monocarboxylate transporter (MCT) homologues confirms the existence of a transporter family with an ancient past. Biochem $J$ 329: 321-328.

Raldúa D, André M, Babin PJ. 2008. Clofibrate and gemfibrozil induce an embryonic malabsorption syndrome in zebrafish. Toxicol Appl Pharmacol 228: 301-314.

Rudel LL, Morris MD. 1973. Determination of cholesterol using o-phthalaldehyde. J Lipid Res 14: 364-366.

Sadler KC, Amsterdam A, Soroka C, Boyer J, Hopkins N. 2005. A genetic screen in zebrafish identifies the mutants vps18, nf 2 and foie gras as models of liver disease. Development 132: 3561-3572.

Sanyal AJ, Chalasani N, Kowdley KV, McCullough A, Diehl AM, Bass NM, Neuschwander-Tetri BA, Lavine JE, Tonascia J, Unalp A, et al. 2010. Pioglitazone, vitamin E, or placebo for nonalcoholic steatohepatitis. N Engl J Med 362: 16751685.

Schlegel A, Stainier DY. 2006. Microsomal triglyceride transfer protein is required for yolk lipid utilization and absorption of dietary lipids in zebrafish larvae. Biochemistry 45: 1517915187. 
Schlombs K, Wagner T, Scheel J. 2003. Site-1 protease is required for cartilage development in zebrafish. Proc Natl Acad Sci 100: 14024-14029.

Schnitzler K, Küster M, Methfessel C, Fejtl M. 2003. The robocyte: Automated cDNA/mRNA injection and subsequent TEVC recording on Xenopus oocytes in 96-well microtiter plates. Receptors Channels 9: 41-48.

Sengupta S, Peterson TR, Laplante M, Oh S, Sabatini DM. 2010. mTORC1 controls fasting-induced ketogenesis and its modulation by ageing. Nature 468: 1100-1104.

Snyder F, Stephens N. 1959. A simplified spectrophotometric determination of ester groups in lipids. Biochim Biophys Acta 34: 244-245.

Thakur PC, Stuckenholz C, Rivera MR, Davison JM, Yao JK, Amsterdam A, Sadler KC, Bahary N. 2011. Lack of de novo phosphatidylinositol synthesis leads to endoplasmic reticulum stress and hepatic steatosis in cdipt-deficient zebrafish. Hepatology 54: 452-462.

Thisse C, Thisse B. 2008. High-resolution in situ hybridization to whole-mount zebrafish embryos. Nat Protoc 3: 59-69.

Veech RL. 2004. The therapeutic implications of ketone bodies: The effects of ketone bodies in pathological conditions: Ketosis, ketogenic diet, redox states, insulin resistance, and mitochondrial metabolism. Prostaglandins Leukot Essent Fatty Acids 70: 309-319.

Walters KB, Dodd ME, Mathias JR, Gallagher AJ, Bennin DA, Rhodes J, Kanki JP, Look AT, Grinblat Y, Huttenlocher A. 2009. Muscle degeneration and leukocyte infiltration caused by mutation of zebrafish fad24. Dev Dyn 238: 86-99.

Wu JW, Wang SP, Alvarez F, Casavant S, Gauthier N, Abed L, Soni KG, Yang G, Mitchell GA. 2011. Deficiency of liver adipose triglyceride lipase in mice causes progressive hepatic steatosis. Hepatology 54: 122-132. 


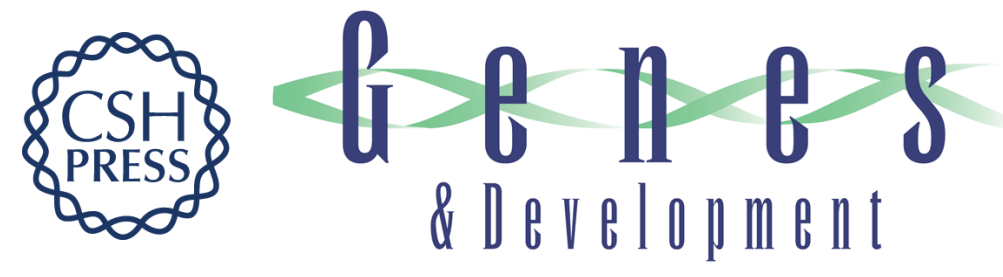

\section{A monocarboxylate transporter required for hepatocyte secretion of ketone bodies during fasting}

Sarah E. Hugo, Lourdes Cruz-Garcia, Santhosh Karanth, et al.

Genes Dev. 2012, 26:

Access the most recent version at doi:10.1101/gad.180968.111

Supplemental http://genesdev.cshlp.org/content/suppl/2012/02/01/26.3.282.DC1
Material

References This article cites 48 articles, 11 of which can be accessed free at: http://genesdev.cshlp.org/content/26/3/282.full.html\#ref-list-1

License

Email Alerting

Receive free email alerts when new articles cite this article - sign up in the box at the top Service right corner of the article or click here.

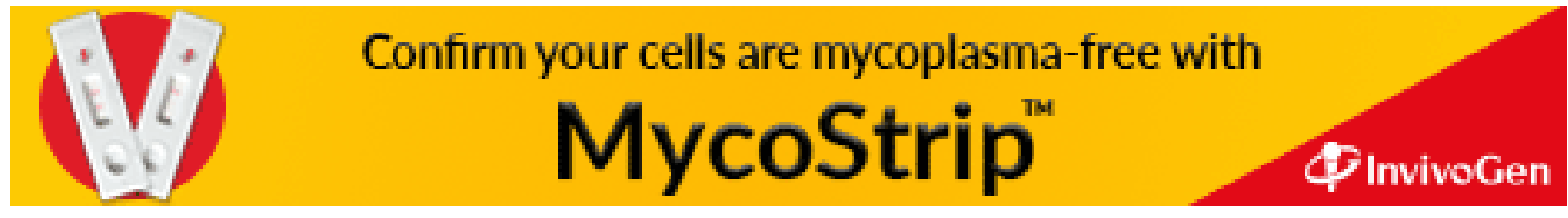

